\title{
Evaluation of Hospital Glycemic Control at US Academic Medical Centers
}

\author{
Jeffrey B. Boord, MD, MPH ${ }^{1,2}$ \\ Robert A. Greevy, PhD ${ }^{1,2}$ \\ Susan S. Braithwaite, $\mathrm{MD}^{3}$ \\ Pamela C. Arnold, RN, MSN ${ }^{4}$ \\ Patricia M. Selig, RN, $\mathrm{PhD}^{5}$ \\ Helga Brake, PharmD ${ }^{6,8}$ \\ Joanne Cuny, RN, MBA ${ }^{6}$ \\ David Baldwin, $\mathrm{MD}^{7}$ \\ ${ }^{1}$ Veterans Affairs (VA) Tennessee Valley Health \\ Care System, Nashville, Tennessee. \\ ${ }^{2}$ Vanderbilt University School of Medicine, Nash- \\ ville, Tennessee. \\ ${ }^{3}$ University of North Carolina School of Medicine, \\ Chapel Hill, North Carolina. \\ ${ }^{4}$ Medical University of South Carolina, Charles- \\ ton, South Carolina. \\ ${ }^{5}$ Virginia Commonwealth University, Richmond, \\ Virginia. \\ ${ }^{6}$ University HealthSystem Consortium, Oakbrook, \\ Illinois. \\ ${ }^{7}$ Rush University Medical Center, Chicago, Illi- \\ nois. \\ ${ }^{8}$ Northwestern Memorial Hospital, Chicago, Illi- \\ nois.
}

OBJECTIVE: To evaluate contemporary hospital glycemic management in US academic medical centers.

DESIGN: This retrospective cohort study was conducted on patients discharged from 37 academic medical centers between July 1 and September 30, 2004; 1,718 eligible adult patients met at least 1 of the inclusion criteria: 2 consecutive blood glucose readings $>180 \mathrm{mg} / \mathrm{dL}$ within 24 hours, or insulin treatment at any time during hospitalization. We assessed 3 consecutive measurement days of glucose values, glycemic therapy, and additional clinical and laboratory characteristics.

RESULTS: In this diverse cohort, $79 \%$ of patients had a prior diagnosis of diabetes, and $84.6 \%$ received insulin on the second measurement day. There was wide variation in hospital performance of recommended hospital diabetes care measures such as glycosylated hemoglobin (A1C) assessment (range, 3\%-63\%) and timely admission laboratory glucose measurement (range, 39\%-97\%). Median glucose was significantly lower for patients in the intensive care unit (ICU) compared to ward/intermediate care. ICU patients treated with intravenous insulin had significantly lower median glucose when compared to subcutaneous insulin. Only $25 \%$ of ICU patients on day 3 had estimated 6 AM glucose $\leq 110 \mathrm{mg} / \mathrm{dL}$. Hyperglycemia was common, $50 \%$ of all patients had $\geq 1$ glucose measurement $\geq 180 \mathrm{mg} / \mathrm{dL}$ on measurement days 2 and 3 . Severe hypoglycemia $(<50 \mathrm{mg} / \mathrm{dL})$ occurred in $2.8 \%$ of all patient days.

CONCLUSIONS: Despite frequent insulin use, glucose control was suboptimal. Academic medical centers have opportunities to improve care to meet current American Diabetes Association hospital diabetes care standards. Journal of Hospital Medicine 2009;4:35-44. (๑ 2009 Society of Hospital Medicine.

KEYWORDS: diabetes mellitus, glycemic control, hospital, insulin therapy.

yperglycemia is a common occurrence in hospitalized patients, with and without a prior diagnosis of diabetes mellitus. $^{1-3}$ Estimates of prevalence of diabetes mellitus in hospitalized adult patients range from $12 \%$ to $25 \%{ }^{4}$ Hyperglycemia is a strong predictor of adverse clinical outcome in a range of diseases such as acute stroke, congestive heart failure, communityacquired pneumonia, and acute myocardial infarction. ${ }^{5-8}$ Hyperglycemia is also a risk factor for surgical infection in patients undergoing cardiac surgery. 9,10 A landmark prospective randomized controlled clinical trial by van den Berghe et al. ${ }^{11}$ demonstrated that tight glucose control (target blood glucose level 80$110 \mathrm{mg} / \mathrm{dL}$ ) with intravenous insulin in critically ill surgical patients led to dramatic reductions in acute renal failure, critical illness polyneuropathy, hospital mortality, and bloodstream infection. Other clinical studies have demonstrated that glycemic control with intravenous insulin improves clinical outcomes and
J.B.B. was supported in part by the Office of Academic Affiliations, Department of Veterans Affairs, VA National Quality Scholars Program, and with resources and facilities at VA Tennessee Valley Healthcare System. Portions of this work were presented in abstract form at the 2006 American Diabetes Association Annual Scientific Sessions. 
reduces length of stay in patients with diabetes undergoing cardiac surgery. ${ }^{12,13}$

Based upon these findings, the American College of Endocrinology (ACE) published recommendations in 2004 for hospital diabetes and metabolic control. ${ }^{14}$ Similar recommendations for hospital glycemic control have been included in the American Diabetes Association (ADA) guidelines since $2005 .{ }^{15}$ There is now emerging consensus that use of continuous insulin infusion given through a standardized protocol is the standard of care to control hyperglycemia in critically ill patients. ${ }^{16-18}$ Likewise, use of specific hospital insulin regimens that include basal and short-acting insulin with appropriate bedside glucose monitoring and avoiding use of "sliding scale" short-acting insulin alone has become recognized as the most effective approach for glucose management in hospitalized patients not requiring intravenous insulin., 19-21

The University HealthSystem Consortium (UHC) is an alliance of 97 academic health centers and 153 of their associated hospitals that conducts benchmarking studies on clinical and operational topics with member academic medical centers and develops new programs to improve quality of care, patient safety, and operational, clinical, and financial performance. In late 2004, UHC launched the Glycemic Control Benchmarking Project to determine the current status of glycemic control in adult patients admitted to academic medical centers, types of treatment employed to control glucose, and operational measures and practices of care for glycemic control in the hospital setting. The goal of the project was to describe contemporary glucose management for the purpose of identifying best practices. The information was later shared with each participating medical center to allow them to better align care delivery with ADA and ACE guidelines. Thirty-seven academic medical centers agreed to participate and submit patient level data as well as an operational survey of current policies and practices for hospital glycemic control. This report summarizes the key findings from retrospective analyses of hospital and patient-level data and describes contemporary management of hyperglycemia in academic medical centers.

\section{PATIENTS AND METHODS}

To be eligible for the study, hospital patients at each participating medical center had to be $\geq 18$ years of age, have a 72-hour or longer length of stay, and be admitted with 1 or more of the following Diagnostic-related group (DRG) codes: 89 (simple pneumonia/ pleurisy), 109 (coronary artery bypass grafting without catheterization), 127 (heart failure and shock), 143 (chest pain), 209 (joint/limb procedure), 316 (renal failure), 478 (other vascular procedures), or 527 (percutaneous intervention with drug eluting stent without acute myocardial infarction). The DRG codes were selected from analysis of the UHC Clinical Data Base because they were the most common adult medical and surgical admission codes that included diabetes as a secondary diagnosis for academic medical centers and were believed to best represent the majority of hospital admissions. Each participating medical center received a secure electronic listing of their eligible patients discharged between July 1, 2004 and September 30, 2004 from the UHC Clinical Data Base. Each center identified data extractors who were trained via teleconference and received technical and content support by UHC staff. The data were collected by chart review and submitted electronically to UHC from February to April 2005.

For each medical center, patients were screened in reverse chronological order proceeding back in time until the minimum number of 50 eligible cases was obtained or until all potential cases were screened. Although 50 cases was the recommended minimum sample size per site, each medical center was encouraged to submit as many eligible cases as possible. The median number of cases submitted by site was 50 (interquartile range [IQR], 42-51). Cases were entered into the study if they met the eligibility criteria and at least one of the following inclusion criteria: (1) two consecutive blood glucose readings $>180 \mathrm{mg} / \mathrm{dL}$ within a $24-$ hour period, or (2) insulin treatment at any time during the hospitalization. Exclusion criteria included history of pancreatic transplant, pregnancy at time of admission, hospice or palliative care during hospital admission, and patients who received insulin for a reason other than blood glucose control (ie, hyperkalemia). Early in the data collection, DRG 209 was dropped from potential screening due to the low yield of meeting screening criteria for blood glucose readings. Of the 315 cases screened for DRG 209 only 44 met all inclusion criteria and remain in the study population.

A maximum of 3 consecutive days of blood glucose (BG) readings were collected for each 
patient, referred to as measurement day 1 , measurement day 2, and measurement day 3. Measurement day 1 is defined as the day the first of 2 consecutive blood glucose levels $>180 \mathrm{mg} / \mathrm{dL}$ occurred during the hospitalization or as the first day insulin was administered during the hospitalization, whichever came first; $40.6 \%$ of patients had the day of admission as their first measurement day. Glucose measurements were recorded by hour for each measurement day as available, and if more than 1 glucose value was available within a particular hour, only the first result was recorded. Both bedside and laboratory serum glucose values were utilized, and glycosylated hemoglobin (A1C) values were included if they were recorded during the hospitalization or within 30 days prior to admission; ${ }^{22} 95.7 \%$ of patients had BG results reported for all 3 measurement days. We defined estimated 6 am glucose for each subject as: the 6 Am glucose if it was available; otherwise the average of the $5 \mathrm{Am}$ and $7 \mathrm{Am}$ glucose values if at least 1 of them was available; otherwise the average of the $4 \mathrm{Am}$ and $8 \mathrm{Am}$ glucose values if at least 1 of them was available. Relevant demographics, medical history, hospitalization details, type and route of insulin administration, and discharge data were also collected. For subcutaneous insulin administration, use of regular, lispro, or aspart insulin was classified as short-acting insulin; use of neutral protamine Hagedorn (NPH), ultralente, or glargine insulin was classified as long-acting insulin. For analysis of glycemic control measures, patient-days in which location or glucose data were not recorded were excluded from analysis. For the analysis comparing subcutaneous versus intravenous insulin treatment on glucose control, patients who received a combination of therapy with subcutaneous and intravenous insulin on the same measurement day were excluded from the analysis (44 patients on day 1, 96 on day 2, and 47 on day 3). For this retrospective analysis, UHC provided a deidentified data set to the authors. The study protocol was reviewed by the Vanderbilt University Institutional Review Board and deemed to be nonhuman subject research since the data set contained no personal or institutional identifiers. Therefore, no informed consent of subjects was required.

Measures of glucose control (median glucose and estimated 6 Am glucose) were analyzed by patient-day, ${ }^{23}$ and were compared by a Wilcoxon rank sum test or an analysis of variance, as indi- cated. $P$ values $<0.05$ were considered significant. To compare effects of intravenous (IV) insulin, subcutaneous long-acting \pm short-acting insulin, and subcutaneous short-acting insulin use alone on glycemic control, mixed effects linear regression modeling for median glucose and mixed effects logistic regression modeling for hyperglycemia and hypoglycemia were used to adjust for fixed effects of age, gender, diabetes status, "all patient refined diagnosis related groups" (APRDRG) severity of illness score, outpatient diabetes treatment, patient location, admission diagnosis, and random effect of hospital site. Separate regression models were performed for measurement days 2 and 3. Statistical analyses were performed with Stata version 8 (Stata Corporation, College Station, TX), R version 2.1.0 (R Foundation for Statistical Computing, Vienna, Austria; www. r-project.org), and SAS version 9 (SAS Institute, Cary, NC).

\section{RESULTS}

Thirty-seven US academic medical centers from 24 states contributed to the analysis. A total of 4,367 cases meeting age, length of stay, and DRG criteria were screened for inclusion in the study; $2,649(60.7 \%)$ screened cases were excluded due to failure to meet inclusion criteria (51\%) or presence of exclusionary conditions (9.7\%); 1,718 (39.3\%) screened cases met all criteria and were included in this analysis. Patient characteristics are summarized in Table 1. A majority of patients (79\%) had a documented history of diabetes, and most of these were classified as type 2 diabetes in the hospital record. Of the patients who were classified as having diabetes on admission, 50.8\% were on some form of outpatient insulin therapy with or without oral diabetes agents. Patients with a diagnosis of diabetes had a median admission glucose of $158 \mathrm{mg} / \mathrm{dL}$ (IQR, 118-221), which was significantly higher than the median admission glucose of $119 \mathrm{mg} / \mathrm{dL}$ (IQR, 100-160) for patients without diabetes $(P<0.001$, rank-sum test).

To determine overall glycemic control for the cohort, median glucose was calculated for each patient, stratified by diabetes status and location for each measurement day (Table 2). Patient-days with a location of emergency department (96 patients on day 1, 6 on day 2, and 2 on day 3 ) and two patients whose location was not defined were 
TABLE 1

Characteristics of Adult Patients in 37 US Academic Medical Centers with Two Consecutive Blood Glucose Values $\geq 180 \mathrm{mg} / \mathrm{dL}$ or Receiving Insulin Therapy

Age (years), median (IQR)

Male

Female

Admission glucose (mg/dL)

Race/Ethnicity

White

Black

Hispanic

Other

Diabetes history

Type 2 diabetes mellitus

Type 1 diabetes mellitus

Unspecified/other diabetes mellitus

No history of diabetes mellitus

Outpatient diabetes treatment

Insulin only

Oral agents only

Insulin and oral agents

No drug therapy

Not documented

Hospitalization DRG

127 Heart failure

109 Coronary artery bypass grafting

316 Renal failure

478 Other vascular procedure

89 Pneumonia

527 Percutaneous intervention with stent

143 Chest pain

209 Joint/limb procedure

Primary insurer

Medicare

Private/commercial

Medicaid

Government

Self-pay

Other/unknown

NOTE: Data are given as median (IQR) or $\mathrm{n}(\%)$

Abbreviation: DRG, diagnosis group; IQR, interquartile range.

excluded from the analysis. Overall, median glucose declined from measurement day 1 to day 3 . For patients with diabetes, median glucose was significantly lower in the intensive care unit (ICU) compared to the general ward or intermediate care for measurement days 1 and 2, but not day 3 . This difference was more pronounced in patients without diabetes, with median glucose significantly lower in the ICU for all 3 measurement days compared to other locations. As expected, median glucose was lower for patients without diabetes compared to patients with diabetes for all measurement days and locations. Hyperglycemia was common; 867 of 1,718 (50\%) patients had at least 1 glucose measurement $\geq 180 \mathrm{mg} / \mathrm{dL}$ on both days 2 and $3 ; 18 \%$ of all patients had a median glucose $\geq 180 \mathrm{mg} / \mathrm{dL}$ on all 3 measurement days. Daily 6 Am glucose was the summary glycemic control measure in the clinical trial by van den Berghe et al., ${ }^{11}$ with goal glucose of 80 to $110 \mathrm{mg} /$ $\mathrm{dL}$ in the intensive treatment group. Since the glycemic target of the American College of Endocrinology Position Statement is $<110 \mathrm{mg} / \mathrm{dL}$ (based largely on van den Berghe et al. ${ }^{11}$ ) we also calculated estimated 6 Am glucose for ICU patient-days to determine the proportion of patients attaining this target. ${ }^{14}$ Estimated 6 AM glucose was lower in ICU patients without diabetes compared to those with diabetes. For patients with diabetes, only $20 \%$ of patients in the ICU had an estimated $6 \mathrm{Am}$ glucose $\leq 110 \mathrm{mg} / \mathrm{dL}$ on measurement day 2 , and only $24 \%$ on day 3 . For patients without diabetes, $27 \%$ and $25 \%$ had an estimated 6 Am glucose $\leq 110$ $\mathrm{mg} / \mathrm{dL}$ on days 2 and 3 , respectively.

For the overall cohort, insulin was the most common treatment for hyperglycemia, with $84.6 \%$ of all patients receiving some form of insulin therapy on the second measurement day. On the second day, $30.8 \%$ received short-acting subcutaneous insulin only, $8.2 \%$ received intravenous insulin infusion, $22.5 \%$ received both short-acting and long-acting subcutaneous insulin, 3.9\% received oral agents, $23 \%$ received some combination of insulin therapies and/or oral agents, and $11.9 \%$ received no treatment. To determine the effect of intravenous versus subcutaneous insulin treatment on glycemic control, we compared patients by insulin treatment and location for each measurement day (Table 3). Intravenous insulin was used predominantly in the ICU, and was associated with significantly lower median glucose compared to subcutaneous insulin in both locations for all 3 measurement days. As expected, the average number of glucose measures per patient was significantly higher for those receiving intravenous insulin. Intravenous insulin use in the ICU was associated with a significantly lower number of patients with hyperglycemia, defined as the number who had 1 or more glucose values $\geq 180$ $\mathrm{mg} / \mathrm{dL}$ during a given measurement day. Of note, intravenous insulin use in the ICU was associated with a significantly higher proportion of patients who had hypoglycemia (defined as the number of patients who had one or more glucose values $<70$ $\mathrm{mg} / \mathrm{dL}$ ) compared to subcutaneous insulin only 
TABLE 2

Glycemic Control Measures for Patients by Diabetes Status, Measurement Day, and Location

\begin{tabular}{|c|c|c|c|}
\hline & \multicolumn{3}{|c|}{ Measurement by Location } \\
\hline & Day 1 & Day 2 & Day 3 \\
\hline \multicolumn{4}{|l|}{ Patients with diabetes } \\
\hline \multicolumn{4}{|c|}{ Estimated 6 AM glucose (mg/dL) } \\
\hline Intensive care unit & $153.0(119.0-204.0)$ & $148.0(118.0-183.0)$ & $144.0(113.0-191.0)$ \\
\hline $\mathrm{n}$ & 167 & 231 & 161 \\
\hline \multicolumn{4}{|c|}{ Median glucose (mg/dL) } \\
\hline General floor & $186.0(151.0-229.0)$ & $163.0(131.0-210.0)$ & $161.0(127.0-203.4)$ \\
\hline $\mathrm{n}$ & 681 & 757 & 758 \\
\hline Intermediate care & $193.0(155.3-233.8)$ & $170.0(137.0-215.5)$ & $169.0(137.9-215.6)$ \\
\hline $\mathrm{n}$ & 291 & 333 & 348 \\
\hline Intensive care unit & $177.5(149.6-213.6)$ & $152.5(128.3-187.0)$ & $156.5(124.5-194.3)$ \\
\hline $\mathrm{n}$ & 294 & 247 & 175 \\
\hline$P$ value* & $0.038^{\dagger}$ & $<0.001^{\dagger}$ & 0.068 \\
\hline \multicolumn{4}{|l|}{ Patients without diabetes } \\
\hline \multicolumn{4}{|c|}{ Estimated 6 AM glucose (mg/dL) } \\
\hline Intensive care unit & $133.0(104.5-174.0)$ & $134.0(109.0-169.0)$ & 128.0 (111.5-151.3) \\
\hline $\mathrm{n}$ & 98 & 157 & 80 \\
\hline \multicolumn{4}{|c|}{ Median glucose (mg/dL) } \\
\hline General floor & $179.0(149.5-209.5)$ & $161.3(131.4-188.3)$ & $143.5(122.0-170.0)$ \\
\hline $\mathrm{n}$ & 91 & 96 & 133 \\
\hline Intermediate care & $168.3(138.1-193.8)$ & $137.0(119.8-161.5)$ & $129.3(116.3-145.5)$ \\
\hline $\mathrm{n}$ & 46 & 71 & 86 \\
\hline Intensive care unit & $153.8(132.9-188.8)$ & $136.5(120.0-157.0)$ & $129.0(116.0-143.8)$ \\
\hline $\mathrm{n}$ & 218 & 186 & 106 \\
\hline$P$ value* & $<0.001^{\dagger}$ & $<0.001^{\dagger}$ & $<0.001^{\dagger}$ \\
\hline \multicolumn{4}{|c|}{ NOTE: Data are median (IQR) or $\mathrm{n}$. } \\
\hline \multicolumn{4}{|c|}{ Abbreviation: IQR, interquartile range. } \\
\hline \multicolumn{4}{|c|}{${ }^{*} P$ value obtained by analysis of variance. } \\
\hline Intensive care unit significant & ther locations by nairui & & \\
\hline
\end{tabular}

on measurement day $1(8.1 \%$ versus $2.9 \% ; P=$ $0.021)$, but not on days $2(12.7 \%$ versus $8.0 \%$; $P>$ $0.05)$ or $3(12.7 \%$ versus $7.8 \% ; P>0.05)$. Severe hypoglycemia, defined as a blood glucose recording $<50 \mathrm{mg} / \mathrm{dL}{ }^{24}$ was rare, and occurred in only $2.8 \%$ of all patient days. On measurement day 1 , 34 patients had a total of 49 severe hypoglycemic events; on day 2, 54 patients had 68 severe hypoglycemic events; on day 3, 54 patients had 68 severe hypoglycemic events. Only 3 patients had severe hypoglycemic events on all 3 measurement days. Analysis of severe hypoglycemia events stratified by intravenous versus subcutaneous insulin did not show any significant differences for any of the 3 measurement days (data not shown).

We hypothesized that use of subcutaneous long-acting (basal) insulin (with or without shortacting insulin) would be associated with superior glucose control compared to use of subcutaneous short-acting insulin (sliding scale and/or sched- uled prandial insulin) alone. We performed an exploratory multivariate regression analysis to compare the effect of IV insulin, long acting subcutaneous insulin \pm short acting insulin, or short acting subcutaneous insulin alone on median glucose, hyperglycemic events (glucose $\geq 180 \mathrm{mg} / \mathrm{dL}$ ), and hypoglycemic events (glucose $<70 \mathrm{mg} / \mathrm{dL}$ ) for days 2 and 3 (Table 4). Compared to short-acting subcutaneous insulin alone, use of IV insulin but not long-acting subcutaneous insulin was predictive of lower median glucose for days 2 and 3. Use of long-acting subcutaneous insulin was not associated with significantly lower odds of hyperglycemic events for days 2 and 3, but was associated with higher odds of hypoglycemic events on day 2 (odds ratio $[\mathrm{OR}], 1.8 ; P=0.01$ ) when compared to short-acting subcutaneous insulin alone.

We measured the performance of recommended hospital diabetes care practices (A1C assessment, documentation of diabetes history in 
TABLE 3

Median Glucose (in mg/dL) by Insulin Treatment Type, Location, and Day

\begin{tabular}{|c|c|c|c|c|}
\hline Location/Day & Outcome & Intravenous Insulin & Subcutaneous Insulin & $P$ Value* \\
\hline \multirow[t]{6}{*}{ Intensive Care Unit, Day 1} & Patient's glucose, median $(\mathrm{mg} / \mathrm{dL})$ & 148.0 & 183.0 & $<0.001$ \\
\hline & Interquartile range & $128.0-178.0$ & $154.8-211.0$ & \\
\hline & Hypoglycemic patients, n (\%) & $16(8.1)$ & $6(2.9)$ & 0.021 \\
\hline & Hyperglycemic patients, n (\%) & $130(66.0)$ & $175(85.0)$ & $<0.001$ \\
\hline & Average glucose measures/patient & 8.4 & 4.8 & $<0.001$ \\
\hline & Patients, $\mathrm{n}$ & 197 & 206 & \\
\hline \multirow[t]{6}{*}{ Intermediate/General Ward, Day 1} & Patient's glucose, median $(\mathrm{mg} / \mathrm{dL})$ & 152.0 & 186.5 & $<0.001$ \\
\hline & Interquartile range & $131.0-164.5$ & $150.0-230.0$ & \\
\hline & Hypoglycemic patients, n (\%) & $1(4.1)$ & $71(7.4)$ & ns \\
\hline & Hyperglycemic patients, n (\%) & $18(78.3)$ & $808(83.9)$ & ns \\
\hline & Average glucose measures/patient & 9.7 & 3.8 & $<0.001$ \\
\hline & Patients, $\mathrm{n}$ & 23 & 962 & \\
\hline \multirow[t]{6}{*}{ Intensive Care Unit, Day 2} & Patient's glucose, median $(\mathrm{mg} / \mathrm{dL})$ & 124.8 & 159.8 & $<0.001$ \\
\hline & Interquartile range & $110.4-140.5$ & $138.6-197.4$ & \\
\hline & Hypoglycemic patients, n (\%) & $15(12.7)$ & $14(8.0)$ & ns \\
\hline & Hyperglycemic patients, n (\%) & $53(44.9)$ & $135(76.7)$ & $<0.001$ \\
\hline & Average glucose measures/patient & 12.5 & 5.3 & $<0.001$ \\
\hline & Patients, $\mathrm{n}$ & 118 & 176 & \\
\hline \multirow[t]{6}{*}{ Intermediate/General Ward, Day 2} & Patient's glucose, median $(\mathrm{mg} / \mathrm{dL})$ & 136.0 & 168.8 & $<0.001$ \\
\hline & Interquartile range & $116.0-168.0$ & $136.1-215.5$ & \\
\hline & Hypoglycemic patients, n (\%) & $2(6.7)$ & $113(11.3)$ & ns \\
\hline & Hyperglycemic patients, $\mathrm{n}(\%)$ & $18(60.0)$ & $784(78.6)$ & 0.015 \\
\hline & Average glucose measures/patient & 11.0 & 4.6 & $<0.001$ \\
\hline & Patients, $\mathrm{n}$ & 30 & 996 & \\
\hline \multirow[t]{6}{*}{ Intensive Care Unit, Day 3} & Patient's glucose, median (mg/dL) & 123.5 & 171.0 & $<0.001$ \\
\hline & Interquartile range & $110.0-137.1$ & $137.3-198.5$ & \\
\hline & Hypoglycemic patients, $n(\%)$ & $7(12.7)$ & $11(7.8)$ & ns \\
\hline & Hyperglycemic patients, n (\%) & $24(43.6)$ & $101(71.1)$ & $<0.001$ \\
\hline & Average glucose measures/patient & 11.4 & 4.8 & $<0.001$ \\
\hline & Patients, $\mathrm{n}$ & 54 & 141 & \\
\hline \multirow[t]{6}{*}{ Intermediate/General Ward, Day 3} & Patient's glucose, median $(\mathrm{mg} / \mathrm{dL})$ & 129.8 & 166.0 & $<0.001$ \\
\hline & Interquartile range & $120.5-142.3$ & $131.5-208.0$ & \\
\hline & Hypoglycemic patients, $n(\%)$ & $3(13.6)$ & $104(9.8)$ & ns \\
\hline & Hyperglycemic patients, n (\%) & $13(59.1)$ & $773(72.7)$ & \\
\hline & Average glucose measures/patient & 10.3 & 4.3 & $<0.001$ \\
\hline & Patients, $\mathrm{n}$ & 22 & 1,055 & \\
\hline
\end{tabular}

NOTE: "Hypoglycemic patients" is the number of patients who had 1 or more glucose values $<70 \mathrm{mg} / \mathrm{dL}$. "Hyperglycemic patients" is the number who had 1 or more glucose values $\geq 180 \mathrm{mg} / \mathrm{dL}$. "Average glucose measures/patient" is the mean number of glucose measurements per patient.

Abbreviation: ns, not significant.

${ }^{*} P$ values are from Wilcoxon rank sum tests comparing intravenous versus subcutaneous insulin treatment.

the hospital record, admission laboratory glucose assessment, bedside glucose monitoring, recommended insulin therapy) ${ }^{14,15}$ for all study patients, and also stratified performance by hospital (Table 5); $98.6 \%$ of all patients with a diagnosis of diabetes had physician documentation of their diabetes status recorded in the hospital record, and there was consistently high performance of this by hospital (Table 5); $77 \%$ of all patients with a history of diabetes had a laboratory blood glucose result recorded within 8 hours of hospital admission, and $81.3 \%$ of patients with a history of diabetes had blood glucose monitored at least 4 times on measurement day 2. Performance by hospital (Table 5) varied widely for glucose monitoring (range, $56.5 \%-95.5 \%$ of patients by hospital) and admission laboratory glucose assessment (range, 39.0\%$97.1 \%$ of patients by hospital).

Of all patients, $31 \%$ had A1C measurement recorded during their hospitalization or within 30 days prior to admission. There was wide variation in hospital performance of AlC assessment in patients with diabetes (Table 5). Patients with a diagnosis of diabetes had a median AlC of 7.4\% (IQR, 
TABLE 4

Regression Analysis of Glycemic Control Measures Comparing Effect of Long-Acting ( \pm Short-Acting) Subcutaneous Insulin and Intravenous Insulin Infusion to Short-Acting Subcutaneous Insulin Alone

\begin{tabular}{|c|c|c|}
\hline Glucose Control Measure & Intravenous Insulin Infusion & Long-Acting Subcutaneous Insulin \\
\hline \multicolumn{3}{|l|}{ Median glucose } \\
\hline Day $2, \mathrm{n}=1,297$ & $-32.0(-45.4$ to -18.5$) ; P<0.001^{*}$ & $-5.1(-13.8$ to 3.6$) ; P=0.25^{*}$ \\
\hline Day $3, \mathrm{n}=1,251$ & $-33.0(-48.9$ to -17$) ; P<0.001^{*}$ & $3.4(-5.2$ to 11.9$) ; P=0.44^{*}$ \\
\hline \multicolumn{3}{|c|}{ Patient has $\geq 1$ hyperglycemic event ${ }^{\dagger}$} \\
\hline Day $2, \mathrm{n}=1,298$ & $0.4(0.2-0.6) ; P<0.001^{\dagger}$ & $0.7(0.5-1.1) ; P=0.11^{\ddagger}$ \\
\hline Day $3, \mathrm{n}=1,261$ & $0.6(0.3-1.1) ; P=0.11^{*}$ & $0.8(0.6-1.1) ; P=0.24$ \\
\hline \multicolumn{3}{|c|}{ Patient has $>1$ hypoglycemic event ${ }^{\S}$} \\
\hline Dav $2, \mathrm{n}=1,298$ & $2.1(1.0-4.7) ; P=0.07^{\star}$ & $1.8(1.2-2.9) ; P=0.010^{*}$ \\
\hline Day $3, \mathrm{n}=1,261$ & $4.0(1.6-9.8) ; P=0.003^{\ddagger}$ & $1.4(0.9-2.3) ; P=0.13^{\ddagger}$ \\
\hline \multicolumn{3}{|c|}{$\begin{array}{l}\text { NOTE: Mixed effects linear regressions for median glucose and mixed effects logistic regressions for hyperglycemia and hypoglycemia were used to adjust for } \\
\text { the effects of location, primary diagnosis, diabetes type, age, gender, preexisting diabetes therapy type, and severity of illness score (all modeled as fixed effects), } \\
\text { and for site (modeled as a random effect). Separate regression models were performed for measurement days } 2 \text { and } 3 \text {. }\end{array}$} \\
\hline \multicolumn{3}{|c|}{ *Values are mean difference $(95 \% \mathrm{CI})$ and $P$ value. Mean difference is in median glucose in $\mathrm{mg} / \mathrm{dL}$ compared to short-acting insulin monotherapy. } \\
\hline \multicolumn{3}{|c|}{${ }^{\dagger}$ Hyperglycemic event is defined as 1 or more glucose values $\geq 180 \mathrm{mg} / \mathrm{dL}$. } \\
\hline \multicolumn{3}{|c|}{ Values are $\mathrm{OR}(95 \% \mathrm{CI})$ and $P$ value. } \\
\hline \multicolumn{3}{|c|}{${ }^{\S}$ Hypoglycemic event is defined as 1 or more glucose values $<70 \mathrm{mg} / \mathrm{dL}$. } \\
\hline Abbreviation: $\mathrm{OR}$, odds ratio; CI & & \\
\hline
\end{tabular}

TABLE 5

Hospital Performance of Recommended Diabetes Care Measures for 37 US Academic Medical Centers

\begin{tabular}{|c|c|c|c|}
\hline Diabetes Care Measure & $\begin{array}{l}\text { Mean Hospital } \\
\text { Performance (\%) }\end{array}$ & $\begin{array}{l}\text { Standard } \\
\text { Deviation (\%) }\end{array}$ & Range (\%) \\
\hline Physician documentation of diabetes history in medical record & 98.8 & 2.1 & $91.5-100$ \\
\hline $\begin{array}{l}\text { AlC assessment documented for diabetes patients (measured during hospitalization or within } 30 \text { days prior } \\
\text { to admission) }\end{array}$ & 33.7 & 15.4 & $3.1-62.9$ \\
\hline Laboratory glucose assessment within 8 hours of hospital presentation for diabetes patients & 77.0 & 13.4 & $39.0-97.1$ \\
\hline Blood glucose monitoring at least 4 times on second measurement day for diabetes patients & 81.6 & 10.8 & $56.5-95.5$ \\
\hline $\begin{array}{l}\text { Percentage of patients receiving insulin therapy who were given short and long-acting insulin OR IV insulin } \\
\text { infusion OR insulin pump therapy on second measurement day }\end{array}$ & 44.9 & 14.3 & $12.1-76.5$ \\
\hline
\end{tabular}

NOTE: Performance for each measure was calculated as number of cases who received the measure divided by total number of cases submitted for that hospital.

Abbreviation: AlC, glycosylated hemoglobin.

$6.4 \%-8.9 \% ; \mathrm{n}=473$ ), and those without a diagnosis of diabetes had a median A1C of $5.9 \%$ (IQR, 5.6\%$6.4 \% ; \mathrm{n}=70$ ). Of the patients with a history of diabetes who had A1C recorded, $59 \%$ had a value $>7 \%$. Of the patients without a history of diabetes who had AlC recorded, $43 \%$ had a value $>6.0 \%$, suggesting previously undiagnosed diabetes. ${ }^{25}$

We found wide variation among hospitals (range, $12.1 \%-76.5 \%$ ) in use of recommended regimens of insulin therapy, defined as short-acting and long-acting subcutaneous insulin or IV insulin infusion or insulin pump therapy on second measurement day. Endocrine/diabetes consultation was infrequent, only $9 \%$ of all patients were evalu- ated by an endocrinologist or diabetologist at any time during the hospitalization.

\section{DISCUSSION}

In this retrospective analysis of hospitalized patients who had 2 consecutive blood glucose values $\geq 180 \mathrm{mg} / \mathrm{dL}$ and/or received insulin therapy, hyperglycemia was common and hypoglycemia was infrequent. Use of intravenous insulin was associated with better glucose control, and did not increase the frequency of severe hypoglycemic events (glucose $<50 \mathrm{mg} / \mathrm{dL}$ ). The majority of patients with a history of diabetes had physician documentation in the hospital chart, labora- 
tory serum glucose obtained within 8 hours of hospital admission, and at least 4 blood glucose determinations on the second measurement day.

Only $35 \%$ of patients with diabetes had an A1C measurement and of these almost $60 \%$ had an AlC level $>7 \%$. Though the AlC may not greatly affect acute glucose management in the hospital setting, it does identify patients that may require intensification of diabetes therapy at hospital discharge and coordination of outpatient follow-up. A report of a UHC clinical benchmarking project of ambulatory diabetes care in academic medical centers demonstrated high rates of diagnostic testing, but only $34 \%$ of patients were at the AlC goal, and only $40 \%$ of patients above the A1C goal had adjustment of their diabetes regimen at their last clinic visit. ${ }^{26}$ In a retrospective study of patients with diabetes mellitus admitted to an academic teaching hospital, only $20 \%$ of discharges indicated a plan for diabetes follow-up. ${ }^{27}$ Thus, intensification of antihyperglycemic therapy and formulation of a diabetes follow-up plan on hospital discharge in those patients with AlC $>7 \%$ represents an opportunity to improve glycemic control in the ambulatory setting. Also, measurement of $\mathrm{AlC}$ can be used for diabetes case-finding in hospitalized patients with hyperglycemia. ${ }^{25}$ Previously unrecognized diabetes is a common finding in patients admitted with cardiovascular disease. In a study of patients admitted with myocardial infarction, $25 \%$ were found to have previously undiagnosed diabetes. ${ }^{28}$ Hospital patients with hyperglycemia but without a prior diagnosis of diabetes who have an elevation of $\mathrm{AlC}>6.0 \%$ can be identified as at-risk for diabetes and postdischarge glucose evaluation can be arranged.

The target of maintaining all glucose values $\leq 180 \mathrm{mg} / \mathrm{dL}$ recommended in the 2005-2007 American Diabetes Association guidelines for hospital diabetes management was not commonly achieved, with over $70 \%$ of patients who received subcutaneous insulin therapy having 1 or more glucose values $>180$ on all 3 measurement days, regardless of patient location. ${ }^{15}$ The target of maintaining critically ill patients as close to 110 $\mathrm{mg} / \mathrm{dL}$ as possible was also difficult to achieve, with only $25 \%$ of ICU patients having an estimated 6 AM glucose $<110 \mathrm{mg} / \mathrm{dL}$ on measurement day 3. A prospective cohort study of 107 inpatients with diabetes at Brigham and Women's Hospital showed a $76 \%$ prevalence of patients with at least one $\mathrm{BG}>180 \mathrm{mg} / \mathrm{dL}^{29}$ In that study, $90 \%$ of patients had a sliding-scale order, $36 \%$ received an oral diabetes agent, and $43 \%$ received basal insulin at some time during hospitalization. A recently published analysis by Wexler et al. $^{30}$ compiled data of hospitalized patients with diabetes from an earlier 2003 UHC Diabetes Benchmarking Project $(n=274)$ and patients from 15 not-for-profit member hospitals of VHA, Incorporated $(n=725)$ to examine the prevalence of hyperglycemia and hypoglycemia. Hyperglycemia (defined as a single $\mathrm{BG}$ value $>200 \mathrm{mg} / \mathrm{dL}$ ) was common, occurring in $77 \%$ of patients in the UHC cohort and $76 \%$ in the VHA, Inc. cohort. This was comparable to our findings that $76.7 \%$ of ICU patients and $78.6 \%$ of ward patients treated with subcutaneous insulin had 1 or more BG values $\geq 180 \mathrm{mg} / \mathrm{dL}$ on measurement day 2 . Wexler et $\mathrm{al}^{30}$ also determined that use of basal insulin was associated with a higher prevalence of hyperglycemia and hypoglycemia in their study. Our regression analysis finding that long-acting (basal) insulin use was not associated with improvement in glycemic control is consistent with the findings of the aforementioned study. There are a number of potential explanations for this: (1) underdosing of basal insulin or lack of adequate prandial insulin coverage for nutritional intake; (2) lack of effective titration in response to hyperglycemia; and (3) variation in the ordering and administration of basal insulin at different hospital sites.

Use of both manual and computerized IV insulin protocols has been shown to provide effective glucose control in critically ill patients. ${ }^{16-18}$ Though intravenous insulin use was associated with better overall glucose control in our study; only about $50 \%$ of ICU patients received it on measurement day 1. A recent prospective randomized clinical trial demonstrated superior glycemic control in non-critically ill hospitalized patients with type 2 diabetes with basal/bolus insulin therapy compared to sliding scale insulin alone. ${ }^{31}$ Use of basal/bolus insulin regimens as part of a comprehensive hospital diabetes management program has been shown to improve glycemic control in an academic medical center. ${ }^{20}$ Therefore, we do not believe that our regression analysis findings invalidate the concept of basal/ bolus insulin for inpatients with hyperglycemia, but rather indicate the need for more research into subcutaneous insulin regimens and hospital care practices that lead to improved glucose control. We found wide variation in hospital use of 
basal/bolus insulin regimens. Overall only $22.5 \%$ of all patients on the second measurement day received both short-acting and long-acting subcutaneous insulin, compared to $30.8 \%$ who received short-acting subcutaneous insulin only. A recent consensus statement on inpatient glycemic control by the American College of Endocrinology and American Diabetes Association highlighted the systematic barriers to improved glycemic control in hospitals, such as inadequate knowledge of diabetes management techniques, fear of hypoglycemia, and skepticism about benefits of tighter glucose control. ${ }^{32}$

There are some important limitations to this study. The data are retrospective and only a limited number of hospital days and clinical variables could be assessed for each patient. As indicated in Table 3, there were significant differences in the frequency of glucose measurement depending on treatment, which can potentially bias estimated prevalence of hyperglycemia and hypoglycemia. We did not have a practical method to assess nutritional status or the adequacy of insulin dosing over time for each patient. We also could not assess the association of glycemic control on clinical outcomes such as hospital mortality or infection rates. Since this study was exclusively in academic medical centers, the generalization of findings to community-based medical centers may be limited. The risk-benefit of tight glycemic control in medical ICU patients based on clinical trial evidence has been unclear, and there is not broad agreement among clinicians on the recommended target for glycemic control in this group. ${ }^{33-35}$ When we analyzed glycemic control in ICU patients we did not have a practical method to control for type of ICU and variations in individual ICU glycemic control targets. We recognize that the 2004 American College of Endocrinology recommendation of maintaining glucose $\leq 110 \mathrm{mg} / \mathrm{dL}$ may not be appropriate for all critically ill patients. ${ }^{14}$ Finally, clinical trial data are lacking on the effect of tight glucose control on major clinical outcomes for non-critically ill hospital patients. This has led to significant controversy regarding glycemic targets for different subgroups of hospitalized patients. ${ }^{34,36}$

In summary, we found a high prevalence of persistent hyperglycemia in this large cohort of hospitalized patients, and hypoglycemia was infrequent. Use of IV insulin was associated with improvement in glycemic control, but was used in less than half of ICU patients. There was wide var- iation in hospital performance of recommended diabetes care measures. Opportunities to improve care in academic medical centers include expanded use of intravenous and subcutaneous basal/bolus insulin protocols and increased frequency of AlC testing.

Address for correspondence and reprint requests: Jeffrey B. Boord, MD, MPH, Vanderbilt University Division of Cardiovascular Medicine, MCE South Tower Suite 5209, 1215 21st Avenue South, Nashville, TN 37232-8802; Telephone: 615-322-2318; Fax: 615-343-4841; E-mail: jeffrey.boord@vanderbilt.edu

Received 8 0ctober 2007; revision received 20 April 2008; accepted 23 April 2008.

\section{REFERENCES}

1. Williams LS, Rotich J, Qi R, et al. Effects of admission hyperglycemia on mortality and costs in acute ischemic stroke. Neurology. 2002;59:67-71.

2. Umpierrez GE, Isaacs SD, Bazargan N, You X, Thaler LM, Kitabchi AE. Hyperglycemia: an independent marker of inhospital mortality in patients with undiagnosed diabetes. J Clin Endocrinol Metab. 2002;87:978-982.

3. Levetan CS, Passaro M, Jablonski K, Kass M, Ratner RE. Unrecognized diabetes among hospitalized patients. Diabetes Care. 1998;21:246-249.

4. Clement S, Braithwaite SS, Magee MF, et al. Management of diabetes and hyperglycemia in hospitals. Diabetes Care. 2004;27:553-591.

5. Norhammar AM, Ryden L, Malmberg K. Admission plasma glucose. Independent risk factor for long-term prognosis after myocardial infarction even in nondiabetic patients. Diabetes Care. 1999;22:1827-1831.

6. Capes SE, Hunt D, Malmberg K, Pathak P, Gerstein HC. Stress hyperglycemia and prognosis of stroke in nondiabetic and diabetic patients: a systematic overview. Stroke. 2001;32:2426-2432.

7. Capes SE, Hunt D, Malmberg K, Gerstein HC. Stress hyperglycaemia and increased risk of death after myocardial infarction in patients with and without diabetes: a systematic overview. Lancet. 2000;355:773-778.

8. McAlister FA, Majumdar SR, Blitz S, Rowe BH, Romney J, Marrie TJ. The relation between hyperglycemia and outcomes in 2,471 patients admitted to the hospital with communityacquired pneumonia. Diabetes Care. 2005;28:810-815.

9. Trick WE, Scheckler WE, Tokars JI, et al. Modifiable risk factors associated with deep sternal site infection after coronary artery bypass grafting. J Thorac Cardiovasc Surg. 2000;119:108-114.

10. Latham R, Lancaster AD, Covington JF, Pirolo JS, Thomas $\mathrm{CS}$. The association of diabetes and glucose control with surgical-site infections among cardiothoracic surgery patients. Infect Control Hosp Epidemiol. 2001;22:607-612.

11. van den Berghe G, Wouters P, Weekers F, et al. Intensive insulin therapy in the critically ill patients. $N$ Engl J Med. 2001;345:1359-1367.

12. Lazar HL, Chipkin SR, Fitzgerald CA, Bao Y, Cabral H, Apstein CS. Tight glycemic control in diabetic coronary artery bypass graft patients improves perioperative outcomes and decreases recurrent ischemic events. Circulation. 2004;109:1497-1502. 
13. Furnary AP, Wu Y, Bookin SO. Effect of hyperglycemia and continuous intravenous insulin infusions on outcomes of cardiac surgical procedures: the Portland Diabetic Project. Endocr Pract. 2004;10(Suppl 2):21-33.

14. Garber AJ, Moghissi ES, Bransome ED Jr, et al. American College of Endocrinology position statement on inpatient diabetes and metabolic control. Endocr Pract. 2004; 10(Suppl 2):4-9.

15. American Diabetes Association. Standards of medical care in diabetes. Diabetes Care. 2005;28(Suppl 1):S4-S36.

16. Goldberg PA, Siegel MD, Sherwin RS, et al. Implementation of a safe and effective insulin infusion protocol in a medical intensive care unit. Diabetes Care. 2004;27: 461-467.

17. Rood E, Bosman RJ, van der Spoel JI, Taylor P, Zandstra DF. Use of a computerized guideline for glucose regulation in the intensive care unit improved both guideline adherence and glucose regulation. J Am Med Inform Assoc. 2005;12: $172-180$.

18. Boord JB, Sharifi M, Greevy RA, et al. Computer-based insulin infusion protocol improves glycemia control over manual protocol. J Am Med Inform Assoc. 2007;14: 278-287.

19. Golightly LK, Jones MA, Hamamura DH, Stolpman NM, McDermott MT. Management of diabetes mellitus in hospitalized patients: efficiency and effectiveness of sliding-scale insulin therapy. Pharmacotherapy. 2006;26:1421- 1432.

20. Baldwin D, Villanueva G, McNutt R, Bhatnagar S. Eliminating inpatient sliding-scale insulin: a reeducation project with medical house staff. Diabetes Care. 2005;28: 1008-1011.

21. Schoeffler JM, Rice DA, Gresham DG. 70/30 insulin algorithm versus sliding scale insulin. Ann Pharmacother. 2005;39:1606-1610.

22. Dungan K, Chapman J, Braithwaite SS, Buse J. Glucose measurement: confounding issues in setting targets for inpatient management. Diabetes Care. 2007;30:403-409.

23. Goldberg PA, Bozzo JE, Thomas PG, et al. "Glucometrics"-assessing the quality of inpatient glucose management. Diabetes Technol Ther. 2006;8:560-569.

24. American Diabetes Association. Hospital admission guidelines for diabetes. Diabetes Care. 2004;27(Suppl 1):S103.
25. Greci LS, Kailasam M, Malkani S, Katz DL, Hulinsky I, Ahmadi R, Nawaz H. Utility of HbA(1c) levels for diabetes case finding in hospitalized patients with hyperglycemia. Diabetes Care. 2003;26:1064-1068.

26. Grant RW, Buse JB, Meigs JB. Quality of diabetes care in U.S. academic medical centers: low rates of medical regimen change. Diabetes Care. 2005;28:337-442.

27. Knecht LA, Gauthier SM, Castro JC, et al. Diabetes care in the hospital: is there clinical inertia? J Hosp Med. 2006; 1:151-160.

28. Norhammar A, Tenerz A, Nilsson G, et al. Glucose metabolism in patients with acute myocardial infarction and no previous diagnosis of diabetes mellitus: a prospective study. Lancet. 2002;359:2140-2144.

29. Schnipper JL, Barsky EE, Shaykevich S, Fitzmaurice G, Pendergrass ML. Inpatient management of diabetes and hyperglycemia among general medicine patients at a large teaching hospital. J Hosp Med. 2006;1:145-150.

30. Wexler DJ, Meigs JB, Cagliero E, Nathan DM, Grant RW. Prevalence of hyper- and hypoglycemia among inpatients with diabetes: a national survey of 44 U.S. hospitals. Diabetes Care. 2007;30:367-369.

31. Umpierrez GE, Smiley D, Zisman A, et al. Randomized study of basal-bolus insulin therapy in the inpatient management of patients with type 2 diabetes (RABBIT 2 trial). Diabetes Care. 2007;30:2181-2186.

32. The ACE/ADA Task Force on Inpatient Diabetes. American College of Endocrinology and American Diabetes Association Consensus Statement on Inpatient Diabetes and Glycemic Control: a call to action. Diabetes Care. 2006;29:1955-1962.

33. van den Berghe G, Wilmer A, Hermans G, et al. Intensive insulin therapy in the medical ICU. $N$ Engl J Med. 2006; 354:449-461.

34. Vanhorebeek I, Langouche L, van den Berghe G. Tight blood glucose control with insulin in the ICU: facts and controversies. Chest. 2007;132:268-278.

35. Brunkhorst FM, Engel C, Bloos F, et al. Intensive insulin therapy and pentastarch resuscitation in severe sepsis. N Engl J Med. 2008;358:125-139.

36. Inzucchi SE, Rosenstock J. Counterpoint: Inpatient glucose management: a premature call to arms? Diabetes Care. 2005;28:976-979. 